\title{
UNIFORMLY QUASIREGULAR MAPS WITH TOROIDAL JULIA SETS
}

\author{
RIIKKA KANGASLAMPI, KIRSI PELTONEN, AND JANG-MEI WU
}

\begin{abstract}
The iterates of a uniformly quasiregular map acting on a Riemannian manifold are quasiregular with a uniform bound on the dilatation. There is a Fatou-Julia type theory associated with the dynamical system obtained by iterating these mappings. We construct the first examples of uniformly quasiregular mappings that have a 2-torus as the Julia set. The spaces supporting this type of mappings include the Hopf link complement and its lens space quotients.
\end{abstract}

\section{INTRODUCTION}

We construct uniformly quasiregular mappings on Riemannian manifolds that have a 2-torus as the Julia set. The spaces supporting these mappings include the Hopf link complement in $\mathbb{S}^{3}$ and its lens space quotients, equipped with Semmestype metrics.

Semmes' idea of creating new metrics on subsets of Euclidean spaces relating the geometry of the metrics to the topological characteristics of the sets was introduced to provide counterexamples to some natural conjectures on the bi-Lipschitz and quasisymmetric parametrizations of metric 3-spheres (see [11] and [12]). This approach has been applied, for example, in 2 to study spaces that are bi-Lipschitz inequivalent to the standard $\mathbb{S}^{3}$ and nevertheless admit maps of bounded length distortion onto $\mathbb{S}^{3}$; in $[9$, to prove a sharp non-Euclidean Picard-type theorem and, in [3], to construct geometrically nice metric $n$-spheres in dimension $n \geq 4$ that do not admit quasisymmetric parametrization by $\mathbb{S}^{n}$.

A continuous mapping $f \in W_{l o c}^{1, n}$ between two oriented Riemannian $n$-manifolds $M$ and $N$ is $K$-quasiregular if it satisfies the distortion inequality

$$
|D f|^{n} \leq K J_{f} \text { a.e. in } M,
$$

where $|D f|$ is the operator norm of the differential $D f$ and $J_{f}$ is the Jacobian determinant of $D f$. A non-injective mapping $f: M \rightarrow M$ is called uniformly quasiregular (uqr) if there exists a constant $1 \leq K \leq \infty$ such that all the iterates $f^{k}$ are $K$-quasiregular with distortion independent of the number of iterates.

Received by the editors October 10, 2011.

2010 Mathematics Subject Classification. Primary 53A30, 53C20; Secondary 30C65.

Key words and phrases. Uniformly quasiregular mapping, Lattès-type mapping, Julia set, conformal structure, lens space.

The first author was supported by the Emil Aaltonen Foundation.

The second author was supported by the Väisälä Foundation of the Finnish Academy of Science and Letters.

The third author was supported by the National Science Foundation Grant DMS-1001669. 
The Fatou set $\mathcal{F}_{f}$ of the uniformly quasiregular mapping $f: M \rightarrow M$ is the set where the family of iterates $\left\{f^{k} \mid k=1,2, \ldots\right\}$ is normal. That is,

$$
\begin{aligned}
\mathcal{F}_{f}=\{x \in M: \text { there exists an open set } U \subset M \\
\text { such that } \left.x \in U \text { and }\left\{\left.f^{k}\right|_{U}\right\} \text { is normal }\right\} .
\end{aligned}
$$

The Julia set $\mathcal{J}_{f}$ of the uniformly quasiregular mapping $f$ is the complement of its Fatou set. We denote by $B_{f}$ the branch set of a quasiregular mapping $f$. It consists of those points in the domain of $f$ where the mapping is not locally injective.

A theorem stated in [4, Theorem 21.4.1] summarizes a procedure in constructing a certain type of uqr maps. The proof of Iwaniec and Martin in [4] is written for the case $M=\overline{\mathbb{R}}^{n}$, but it holds more generally on a Riemannian manifold $M$ without changes.

Theorem 1.1. Let $\Gamma$ be a discrete group of isometries of $\mathbb{R}^{n}$, and $h: \mathbb{R}^{n} \rightarrow M$ be a quasiregular mapping that is automorphic with respect to $\Gamma$ in the strong sense. If there is a similarity $A=\lambda \mathcal{O}, \lambda \in \mathbb{R}, \lambda \neq 0$, and $\mathcal{O}$ an orthogonal transformation, such that

$$
A \Gamma A^{-1} \subset \Gamma
$$

then there is a unique solution $f: h\left(\mathbb{R}^{n}\right) \rightarrow h\left(\mathbb{R}^{n}\right)$ to the Schröder functional equation

$$
f \circ h=h \circ A,
$$

and $f$ is a uniformly quasiregular mapping.

In the above theorem we use the following definition for a mapping that is automorphic in the strong sense.

Definition 1.2. Let $\Gamma$ be a discrete group of isometries of $\mathbb{R}^{n}$. A mapping $h$ : $\mathbb{R}^{n} \rightarrow M$ is automorphic with respect to $\Gamma$ in the strong sense if

(1) $h \circ \gamma=h$ for any $\gamma \in \Gamma$,

(2) $\Gamma$ acts transitively on the fibres $\mathcal{O}_{y}=h^{-1}(y)$.

Note that following from (1.1) we have the equation $f^{k} \circ h=h \circ A^{k}$ for all $k$. Thus the dilatation of the uqr mapping $f^{k}$ is at most the dilatation of $h^{2}$ for all $k=1,2, \ldots$

We call the mappings arising from Theorem 1.1 uniformly quasiregular mappings of Lattès type. This type of mappings acting on the $n$-sphere has been studied by Volker Mayer in [7] and 8]. In fact, he showed that in addition to the so-called chaotic rational maps [5] there exist analogues of planar power mappings $z \mapsto z^{d}$ as well as Tchebychev polynomials such as $z \mapsto z^{2}-2$. In this respect the theory of uqr maps on higher dimensional spheres is equally rich as in two dimensions. The uniformly quasiregular counterparts for power mappings have a codimension 1 sphere as a Julia set with origin and infinity as super-attracting and completely invariant fixed points. The uqr counterpart of Tchebychev polynomials have a codimension 1 closed unit disk as a Julia set and completely invariant fixed point infinity. In [1] a variety of uqr maps of Lattès type are constructed acting on closed Riemannian manifolds, including spaces that can be products of spheres, tori and odd dimensional real projective spaces. Typically these maps are chaotic, but in some cases the maps produced there have interesting Julia sets. For example, every odd dimensional projective space supports a uqr map which has a codimension 
one (non-orientable!) real projective space as a Julia set. This is possible in the presence of exactly one superattractive fixed point and one basin of attraction.

We do not know whether lens spaces $L(p, q)$, where $p>q>0$ are coprime integers and $p \geq 3$, support Lattès type mappings. G. Martin and the second author have shown that the 2-torus cannot appear as a Julia set either on the standard $\mathbb{S}^{3}$ or on a lens space $L(p, q)$ equipped with the quotient metric [6]. The book of Rolfsen [10] contains illustrated descriptions of lens spaces.

\section{UQR MAPPING WITH TOROIDAL JULIA SET}

First we formulate the main theorem of this section.

Theorem 2.1. Let $H$ be a Hopf link. There exists a Riemannian metric $g_{H}$ on $\mathbb{S}^{3} \backslash H$ and a uniformly quasiregular map $f:\left(\mathbb{S}^{3} \backslash H, g_{H}\right) \rightarrow\left(\mathbb{S}^{3} \backslash H, g_{H}\right)$ of Lattès type such that the branch set $B_{f}=\emptyset$ and the Julia set of $f$ is a 2-torus.

Suppose $H$ is a Hopf link on $\mathbb{S}^{3}$. P. Pankka and K. Rajala proved in [9] that the manifold $\mathbb{S}^{3} \backslash H$ may be equipped with a Riemannian metric $g_{H}$ so that it is quasiregularly elliptic. That is, there exists a quasiregular mapping $h: \mathbb{R}^{3} \rightarrow$ $\left(\mathbb{S}^{3} \backslash H, g_{H}\right)$. In fact it is even possible to choose this metric in such a way that the mapping $h$ is conformal.

With the help of this mapping $h$ and the metric $g_{H}$, we define a uniformly quasiregular map $f \equiv f_{\lambda}$ of Lattès type on $\left(\mathbb{S}^{3} \backslash H, g_{H}\right)$, for an arbitrary dilation $A_{\lambda}: \mathbb{R}^{3} \rightarrow \mathbb{R}^{3},(x, y, t) \mapsto(\lambda x, \lambda y, \lambda t)$, and an integer $\lambda \geq 2$, so that the following diagram commutes.

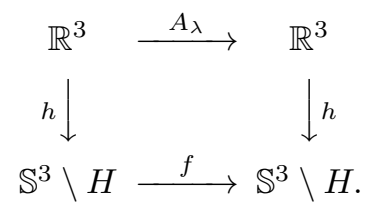

Consider $\mathbb{S}^{3}=\left\{\left.(z, w)|| z\right|^{2}+|w|^{2}=1\right\} \subset \mathbb{C}^{2}$ and the Hopf link $H=S_{0} \cup S_{1}$ with $S_{0}=\left\{\left(0, \mathrm{e}^{2 \pi \mathrm{i} y}\right) \mid y \in(0,1]\right\}$ and $S_{1}=\left\{\left(\mathrm{e}^{2 \pi \mathrm{i} x}, 0\right) \mid x \in(0,1]\right\}$. Define mapping $h: \mathbb{R}^{3} \rightarrow \mathbb{S}^{3} \backslash H$ by setting

$$
h(x, y, t)=\left(\alpha(t) \mathrm{e}^{2 \pi \mathrm{i} x}, \beta(t) \mathrm{e}^{2 \pi \mathrm{i} y}\right),
$$

where $\alpha, \beta: \mathbb{R} \rightarrow(0,1)$ are $C^{\infty}$ smooth diffeomorphisms defined by conditions

$$
\alpha(t)=\left(\frac{1}{\pi} \arctan t+\frac{1}{2}\right)^{\frac{1}{2}}, \beta(t)=\left(1-\alpha(t)^{2}\right)^{\frac{1}{2}} .
$$

On $\mathbb{R}^{3}$ we consider the Euclidean metric and on $\mathbb{S}^{3} \backslash H$ we consider the pushforward metric induced by a $C^{\infty}$ smooth diffeomorphism $\Phi: \mathbb{S}^{1} \times \mathbb{S}^{1} \times \mathbb{R} \rightarrow$ $\mathbb{S}^{3} \backslash H$ while $\mathbb{S}^{1} \times \mathbb{S}^{1} \times \mathbb{R}$ is equipped with the standard product metric. In the above, the unit circles $\mathbb{S}^{1} \subset \mathbb{C}$ are equipped with the ambient Euclidean metric in the plane and $\mathbb{R}$ is the standard real line, and the mapping $\Phi$ is defined by 
the condition

$$
\left(\mathrm{e}^{2 \pi \mathrm{i} x}, \mathrm{e}^{2 \pi \mathrm{i} y}, t\right) \mapsto\left(\alpha(t) \mathrm{e}^{2 \pi \mathrm{i} x}, \beta(t) \mathrm{e}^{2 \pi \mathrm{i} y}\right)
$$

with functions $\alpha$ and $\beta$ defined earlier. If $g$ is the product metric in $\mathbb{S}^{1} \times \mathbb{S}^{1} \times \mathbb{R}$, the smooth metric $g_{H}$ on $\mathbb{S}^{3} \backslash H$ that makes mapping $\Phi$ an isometry is defined by the condition

$$
\left(g_{H}\right)_{p}(v, w)=g\left(\left((D \Phi)_{\Phi^{-1} p}\right)^{-1} v,\left((D \Phi)_{\Phi^{-1} p}\right)^{-1} w\right),
$$

for $p \in \mathbb{S}^{3} \backslash H$ and $v, w \in T_{p}\left(\mathbb{S}^{3} \backslash H\right)$.

Under the above choice of the metric, the mapping $h: \mathbb{R}^{3} \rightarrow \mathbb{S}^{3} \backslash H$ is a conformal covering map. Especially the branch set of $h$ is empty. Each square

$$
\{(x, y, a) \mid x \in(m, m+1], y \in(n, n+1]\},
$$

$m, n \in \mathbb{Z}$ and $a \in \mathbb{R}$, is mapped bijectively onto a 2 -torus

$$
M_{a}=\left\{\left(\alpha(a) \mathrm{e}^{2 \pi \mathrm{i} x}, \beta(a) \mathrm{e}^{2 \pi \mathrm{i} y}\right) \mid x, y \in(0,1]\right\}
$$

under $h$. The restriction $h \mid \mathbb{R}^{2} \times\{a\}: \mathbb{R}^{2} \times\{a\} \rightarrow M_{a}$ is a conformal covering map of the 2-torus. Furthermore, every semi-infinite cylinder

$$
\{(x, y, t) \mid x \in(m, m+1], y \in(n, n+1], t<a\}
$$

is mapped bijectively onto the complement of the core $S_{0}$ in the solid torus

$$
\left\{\left(\alpha(t) \mathrm{e}^{2 \pi \mathrm{i} x}, \beta(t) \mathrm{e}^{2 \pi \mathrm{i} y}\right) \mid x, y \in(0,1], t<a\right\} ;
$$

and every semi-infinite cylinder

$$
\{(x, y, t) \mid x \in(m, m+1], y \in(n, n+1], t>a\}
$$

is mapped bijectively onto the complement of the core $S_{1}$ in the solid torus

$$
\left\{\left(\alpha(t) \mathrm{e}^{2 \pi \mathrm{i} x}, \beta(t) \mathrm{e}^{2 \pi \mathrm{i} y}\right) \mid x, y \in(0,1], t>a\right\} .
$$

The image of the $x y$-plane under $h$ is the 2 -torus

$$
M_{0}=\left\{\left(\frac{\sqrt{2}}{2} \mathrm{e}^{2 \pi \mathrm{i} x}, \frac{\sqrt{2}}{2} \mathrm{e}^{2 \pi \mathrm{i} y}\right) \mid x, y \in(0,1]\right\}
$$

which divides $\mathbb{S}^{3} \backslash H$ into two parts $T_{0} \backslash S_{0}$ and $T_{1} \backslash S_{1}$, where $T_{0}$ and $T_{1}$ are two solid tori in $\mathbb{S}^{3}$ with a common boundary $M_{0}$.

To see that the mapping $f$ is well defined for any dilation $A_{\lambda}: \mathbb{R}^{3} \rightarrow \mathbb{R}^{3}$, $(x, y, t) \mapsto(\lambda x, \lambda y, \lambda t)$, and an integer $\lambda \geq 2$, we fix a point

$$
\left(z_{0}, w_{0}\right)=\left(\alpha\left(t_{0}\right) \mathrm{e}^{2 \pi \mathrm{i} x_{0}}, \beta\left(t_{0}\right) \mathrm{e}^{2 \pi \mathrm{i} y_{0}}\right) \in \mathbb{S}^{3} \backslash H
$$

with parameters $x_{0}, y_{0} \in(0,1]$ and $t_{0} \in \mathbb{R}$. The set

$$
h^{-1}\left(z_{0}, w_{0}\right)=\left\{\left(x_{0}+m, y_{0}+n, t_{0}\right) \mid m, n \in \mathbb{Z}\right\}
$$

of preimages of $\left(z_{0}, w_{0}\right)$ under $h$ consists of infinitely many points in the plane $\mathbb{R}^{2} \times\left\{t_{0}\right\}$, one in each square defined by the integer lattice. Hence the image of this set under $h \circ A_{\lambda}$ is a single point

$$
\left(\alpha\left(\lambda t_{0}\right) \mathrm{e}^{2 \pi \mathrm{i} \lambda x_{0}}, \beta\left(\lambda t_{0}\right) \mathrm{e}^{2 \pi \mathrm{i} \lambda y_{0}}\right) \in \mathbb{S}^{3} \backslash H
$$

and the mapping $f$ is well defined.

Since $f^{k} \circ h=h \circ A_{\lambda}^{k}$ for every integer $k \geq 1$, the induced map $f$ is uniformly quasiregular on $\mathbb{S}^{3} \backslash H$. Since the mapping $h$ is conformal, the induced mapping $f$ is also conformal with respect to the metric $g_{H}$. 
Denote by $\Gamma$ the group of isometries generated by translations $e_{1}:(x, y, t) \mapsto$ $(x+1, y, t)$ and $e_{2}:(x, y, t) \mapsto(x, y+1, t)$ in $\mathbb{R}^{3}$. Then $A_{\lambda} \Gamma A_{\lambda}^{-1} \subset \Gamma$ and $h$ is automorphic with respect to $\Gamma$ in the strong sense. So $f$ is of Lattès type.

The fact that the Julia set of the mapping $f$ is the torus $M_{0}$ can be seen as follows. The origin is a repelling fixed point for the mapping $A_{\lambda}:(x, y, t) \mapsto$ $(\lambda x, \lambda y, \lambda t)$. Since the orbit $\Gamma((0,0,0))=\{\gamma(0) \mid \gamma \in \Gamma\}=\mathbb{Z}^{2} \times\{0\}$, the set $E=\bigcup_{k>0} A_{\lambda}^{-k} \Gamma((0,0,0))$ is a dense subset of $\mathbb{R}^{2} \times\{0\}$. Hence, $h(E)$ is a dense subset of $M_{0} \subset \mathbb{S}^{3} \backslash H$. We conclude that $\left(f^{k}\right)$ cannot be equicontinuous in a neighbourhood of any point of $M_{0}$. On the other hand, the points $A_{\lambda}^{k}(x, y, t) \in \mathbb{R}^{3}$, $t \neq 0$, tend to infinity as $k \rightarrow \infty$. The corresponding points $f^{k}(h(x, y, t))=$ $\left(\alpha\left(\lambda^{k} t\right) \mathrm{e}^{2 \pi \mathrm{i} \lambda^{k} x}, \beta\left(\lambda^{k} t\right) \mathrm{e}^{2 \pi \mathrm{i} \lambda^{k} y}\right)$ tend to the omitted circle $S_{1}$ when $t>0$, and to the other circle $S_{0}$ when $t<0$. Hence, the Julia set of $f$ is the torus $M_{0}$ and the completely invariant components of the Fatou set of $f$ are the interiors of the sets $T_{0} \backslash S_{0}$ and $T_{1} \backslash S_{1}$. The degree of the mapping $f$ is $\lambda^{2}$, and there is no branching.

Remark 2.2. The mapping $f$ extends to a mapping $f^{*}$ acting on $\mathbb{S}^{3}$, when $\mathbb{S}^{3} \backslash H$ is compactified by gluing the Hopf link $H$ back and we define $f_{\mid H}^{*}=\operatorname{Id}_{H}$. This mapping $f^{*}$ is a topological conjugate of a winding map acting on $\mathbb{S}^{3}$, whose branch set is the Hopf link $H$. These winding maps acting on solid tori are of the form

$$
(r, \varphi, \theta) \mapsto\left(r, k_{1} \varphi, k_{2} \theta\right),
$$

where integers $k_{i} \geq 1, i=1,2$ depend on $\lambda$ and coordinate $r$ measures the distance from the core and angles $\varphi, \theta$ deviation along the core and the meridian of the torus. These mappings are quasiregular (even of bounded length distortion), but not uniformly quasiregular.

\section{UQR MAPPINGS ON LENS SPACES}

Let $L(p, q)$ be a lens space of type $(p, q)$, where $p>q>0$ are two coprime integers $(p \mid q)=1$. Denote by $\pi: \mathbb{S}^{3} \rightarrow L(p, q)$ the standard $p$ to 1 covering projection. Let $H=S_{0} \cup S_{1}$ be the Hopf link and $g_{H}$ be the metric on $\mathbb{S}^{3} \backslash H$ previously defined. Metric $\tilde{g}_{H}$ on $L(p, q) \backslash \pi(H)$ is the one that makes the covering projection $\pi$ a local isometry.

The main theorem of this section is the following.

Theorem 3.1. There exists a uniformly quasiregular map $f_{L}:\left(L(p, q) \backslash \pi(H), \tilde{g}_{H}\right)$ $\rightarrow\left(L(p, q) \backslash \pi(H), \tilde{g}_{H}\right)$ of Lattès type such that the branch set $B_{f_{L}}=\emptyset$ and the Julia set of $f_{L}$ is a 2 -torus.

To describe the lens spaces $L(p, q)$, we again consider its covering space $\mathbb{S}^{3}$ as the unit sphere $\left\{\left.(z, w)|| z\right|^{2}+|w|^{2}=1\right\}$ in complex 2-space $\mathbb{C}^{2}$. Denote by $\tau: \mathbb{S}^{3} \rightarrow \mathbb{S}^{3}$ the $p$-periodic homeomorphism $\tau(z, w)=\left(z e^{2 \pi \mathrm{i} / p}, w e^{2 \pi \mathrm{i} q / p}\right)$. The orbit space of this action is the lens space $L(p, q)$. Here the points $u$ and $v$ on $\mathbb{S}^{3}$ are identified if and only if $u=\tau^{k}(v)$ for some integer $k$. Then $\mathbb{S}^{3}$ is the universal covering space for $L(p, q)$ and $\tau$ is a generator of the cyclic group of covering translations. We denote the $p$ to 1 covering map by $\pi$. The mapping $\tau$ keeps circles $S_{0}$ and $S_{1}$ invariant. The $p$ to 1 image of these circles under $\pi$ consists of two circles: $\pi\left(S_{0}\right)$ is the edge of the lens and $\pi\left(S_{1}\right)$ is a core of the solid torus $\pi\left(T_{1}\right)$. Furthermore, the image $\pi\left(M_{a}\right)$ of any 2-torus $M_{a}$ in (2.2) is again a 2-torus. To see this we note that the torus $M_{a}$ is invariant under the covering translation $\tau$ and can be subdivided into 
$p$ annuli which are permuted by $\tau$. The projection $\pi$ maps these $p$ annuli onto a single 2-torus, namely $\pi\left(M_{a}\right)$.

We show that for any integer $\lambda \geq 2$ the dilation $A_{\lambda}$ descends to a well-defined mapping $f_{L}$ on $L(p, q) \backslash \pi(H)$ such that the following diagram commutes. Let $f$ and $h$ be the mappings defined in the previous section. By an argument similar to that for Theorem 1.1, $f_{L}$ is uniformly quasiregular.

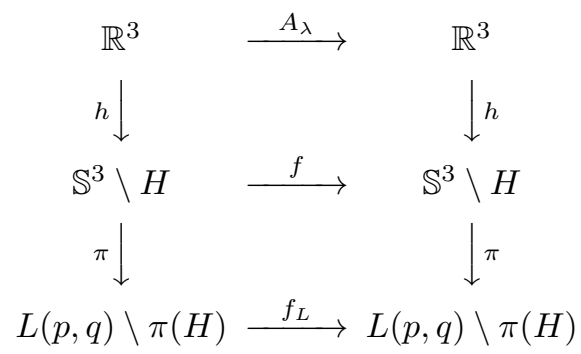

Consider a point $\left[z_{0}, w_{0}\right] \in L(p, q) \backslash \pi(H)$ whose representative in $\mathbb{S}^{3}$ is given by $\left(z_{0}, w_{0}\right)=\left(\alpha \mathrm{e}^{2 \pi \mathrm{i} x_{0}}, \beta \mathrm{e}^{2 \pi \mathrm{i} y_{0}}\right)$ for some $\alpha \in(0,1), \beta=\left(1-\alpha^{2}\right)^{1 / 2}, x_{0}, y_{0} \in(0,1]$. It has $p$ preimage points in $\mathbb{S}^{3} \backslash H$ under the covering map $\pi$ :

$$
\pi^{-1}\left(\left[z_{0}, w_{0}\right]\right)=\left\{\left(\alpha \mathrm{e}^{2 \pi \mathrm{i}\left(x_{0}+\frac{k}{p}\right)}, \beta \mathrm{e}^{2 \pi \mathrm{i}\left(y_{0}+\frac{q k}{p}\right)}\right) \mid k=0,1, \ldots, p-1\right\} .
$$

The preimage of this set under $h$ is contained in the plane $\left\{\left(x, y, t_{\alpha}\right) \mid x, y \in \mathbb{R}\right\}$, where $t_{\alpha}=\tan \left(\pi\left(\alpha^{2}-\frac{1}{2}\right)\right)$. That is

$$
\begin{aligned}
h^{-1} & \left(\pi^{-1}\left(\left[z_{0}, w_{0}\right]\right)\right) \\
& =\left\{\left(x_{0}+\frac{k}{p}+m, y_{0}+\frac{k q}{p}+n, t_{\alpha}\right) \mid k=0,1, \cdots, p-1, m, n \in \mathbb{Z}\right\} .
\end{aligned}
$$

Under $h \circ A_{\lambda}$ the above points are further mapped to points

$$
\begin{aligned}
& h\left(A_{\lambda}\left(h^{-1}\left(\pi^{-1}\left(\left[z_{0}, w_{0}\right]\right)\right)\right)\right) \\
& \quad=\left\{\left(\alpha\left(\lambda t_{\alpha}\right) \mathrm{e}^{2 \pi \mathrm{i} \lambda\left(x_{0}+\frac{k}{p}\right)}, \beta\left(\lambda t_{\alpha}\right) \mathrm{e}^{2 \pi \mathrm{i} \lambda\left(y_{0}+\frac{q k}{p}\right)}\right) \mid k=0,1, \ldots, p-1\right\}
\end{aligned}
$$

on $\mathbb{S}^{3} \backslash H$. Set $(3.2)$ constitutes at most $p$ points. There is only one point if $\lambda$ is a multiple of $p$. However, the projection $\pi$ maps all these points into a single point in $L(p, q) \backslash \pi(H)$, for any integer $\lambda \geq 2$. The mapping $f_{L}:\left[z_{0}, w_{0}\right] \mapsto$ $\left[\alpha\left(\lambda t_{\alpha}\right) e^{2 \pi i \lambda x_{0}}, \beta\left(\lambda t_{\alpha}\right) e^{2 \pi i \lambda y_{0}}\right]$ is well defined, with no restriction on multiplier $\lambda$ for different values of $q$ and $p$.

Since $f_{L}^{k} \circ \pi \circ h=\pi \circ h \circ A_{\lambda}^{k}$ holds for every $k \geq 1$, the mapping $f_{L}$ is uniformly quasiregular. Since $\pi \circ h$ is automorphic with respect to the group $\widetilde{\Gamma}=\{(x, y, t) \mapsto$ $\left.\left(x+\frac{k}{p}+m, y+\frac{k q}{p}+n, t\right) \mid k=0,1, \ldots, p-1, m, n \in Z\right\}$ in the strong sense and $A_{\lambda} \widetilde{\Gamma} A_{\lambda}^{-1} \subset \widetilde{\Gamma}$, map $f_{L}$ is of Lattès type.

By an argument similar to that for the case $\mathbb{S}^{3} \backslash H$, the Julia set of $f_{L}$ is the 2-torus $\pi\left(M_{0}\right)$ that divides the lens space into two parts. On one side of $\pi\left(M_{0}\right)$ all points tend to the circle $\pi\left(S_{0}\right)$ under the iterates, while on the other side, all points tend to the circle $\pi\left(S_{1}\right)$.

Remark 3.2. Note that in the case $\lambda=p$, the mapping $f_{L}$ lifts to a conformal $p$ to 1 covering map $\tilde{f}_{L}: L(p, q) \backslash \pi(H) \rightarrow S^{3} \backslash H$. Namely, for fixed parameters 
$\alpha \in(0,1), \beta=\left(1-\alpha^{2}\right)^{1 / 2}$, and $x_{0}, y_{0} \in(0,1]$, these $p^{2}$ different points

$$
\left\{\left(\alpha \mathrm{e}^{2 \pi \mathrm{i}\left(x_{0}+\frac{k}{p}\right)}, \beta \mathrm{e}^{2 \pi \mathrm{i}\left(y_{0}+\frac{j q}{p}\right)}\right) \mid k=0, \ldots, p-1, j=0, \ldots, p-1\right\}
$$

in $\mathbb{S}^{3} \backslash H$ descent to $p$ different points

$$
\left\{\left[\left(\alpha \mathrm{e}^{2 \pi \mathrm{i}\left(x_{0}+\frac{k}{p}\right)}, \beta \mathrm{e}^{2 \pi \mathrm{i} y_{0}}\right)\right] \mid k=0, \ldots, p-1\right\}
$$

in $L(p, q) \backslash \pi(H)$. By tracking the preimages of these points under $\pi$ and $h$ as above, one gets the following points in $\mathbb{R}^{3}$ :

$$
\left\{\left(x_{0}+\frac{k}{p}+m, y_{0}+\frac{j q}{p}+n, t_{\alpha}\right) \mid k, j=0, \ldots, p-1, m, n \in \mathbb{Z}\right\},
$$

where $t_{\alpha}=\tan \left(\pi\left(\alpha^{2}-\frac{1}{2}\right)\right)$. These points are further mapped under $h \circ A_{p}$ to a single point

$$
\left(\alpha\left(p t_{\alpha}\right) \mathrm{e}^{2 \pi \mathrm{i} p x_{0}}, \beta\left(p t_{\alpha}\right) \mathrm{e}^{2 \pi \mathrm{i} p y_{0}}\right) \in \mathbb{S}^{3} \backslash H .
$$

The covering map $\tilde{f}_{L}$ can be extended to act on the compactified spaces $L(p, q)$ and $\mathbb{S}^{3}$. The extension $\tilde{f}_{L}^{*}: L(p, q) \rightarrow \mathbb{S}^{3}$ is a topological conjugate of a map of bounded length distortion branching along the set $\pi(H)$, but $\tilde{f}_{L}^{*}$ is not uniformly quasiregular.

\section{ACKnowledgment}

The authors are grateful to Pekka Pankka for inspiration and valuable discussions related to this work.

\section{ReFERENCES}

1. Laura Astola, Riikka Kangaslampi, and Kirsi Peltonen, Lattès-type mappings on compact manifolds, Conform. Geom. Dyn. 14 (2010), 337-367. MR2746722

2. Juha Heinonen and Seppo Rickman, Geometric branched covers between generalized manifolds, Duke Math. J. 113 (2002), no. 3, 465-529. MR1909607(2003h:57003)

3. Juha Heinonen and Jang-Mei Wu, Quasisymmetric nonparametrization and spaces associated with the Whitehead continuum, Geom. Topol. 14 (2010), no. 2, 773-798. MR2602851 (2011d:57060)

4. Tadeusz Iwaniec and Gaven Martin, Geometric function theory and non-linear analysis, Oxford Mathematical Monographs, The Clarendon Press Oxford University Press, New York, 2001. MR1859913 (2003c:30001)

5. S. Lattès, Sur l'itération des substitutions rationnelles et les fonctions de Poincarè, C. R. Acad. Sci. Paris 166 (1918), 26-28.

6. Gaven Martin and Kirsi Peltonen, Obstructions for Julia sets in UQR dynamics, In Preparation.

7. Volker Mayer, Uniformly quasiregular mappings of Lattès type, Conform. Geom. Dyn. 1 (1997), 104-111 (electronic). MR1482944 (98j:30017)

8. Q Quasiregular analogues of critically finite rational functions with parabolic orbifold, J. Anal. Math. 75 (1998), 105-119. MR1655826 (2000a:30043)

9. Pekka Pankka and Kai Rajala, Quasiregularly elliptic link complements, Geom. Dedicata 154 (1), 2011. MR2832708

10. Dale Rolfsen, Knots and links, Mathematics Lecture Series, vol. 7, Publish or Perish Inc., Houston, TX, 1990, Corrected reprint of the 1976 original. MR.1277811 (95c:57018) 
11. Stephen Semmes, Good metric spaces without good parametrizations, Rev. Mat. Iberoamericana 12 (1996), no. 1, 187-275. MR 1387590(97e:57025)

12. On the nonexistence of bi-Lipschitz parametrizations and geometric problems about $A_{\infty}$-weights, Rev. Mat. Iberoamericana 12 (1996), no. 2, 337-410. MR1402671 (97e:30040)

Aalto University, P.O. Box 11100, 00076 Aalto, Finland

E-mail address: riikka.kangaslampi@aalto.fi

Aalto University, P.O. Box 11100, 00076 Aalto, Finland

E-mail address: kirsi.peltonen@aalto.fi

Department of Mathematics, University of Illinois, 1409 W. Green Street, Urbana, ILLINOIS 61801-2975

E-mail address: wu@math.uiuc.edu 